\title{
Impact of galvanic vestibular stimulation on mood
}

\section{F. Pasquier, C. Guillot, P. Denise, G. Quarck}

Lab Comete INSERM U1075, Unicaen, Normandie Université, 14032 Caen, France

\section{Introduction}

Galvanic Vestibular Stimulation (GVS) is a variant of transcranial direct current stimulation (tDCS). GVS is applied through electrodes placed over the mastoid. GVS activates primary otolithic and semi-circular canal neurons.

This stimulation in human induces oculomotor, perceptual and postural responses. Several studies have explored the effect of tDCS on mood and particulary on depression. Emerging evidence suggests that vestibular network expands into dimensions of emotion processing, mental health, and social cognition. Our hypothesis is that GVS and tDCS, by sharing a common process, could have certain common impacts.

\section{Feasability study}

- Participants :

- 6 participants in healthy condition were recruited.

- criteria of inclusion : absence of rhythmicity, mood, vestibular disorders.

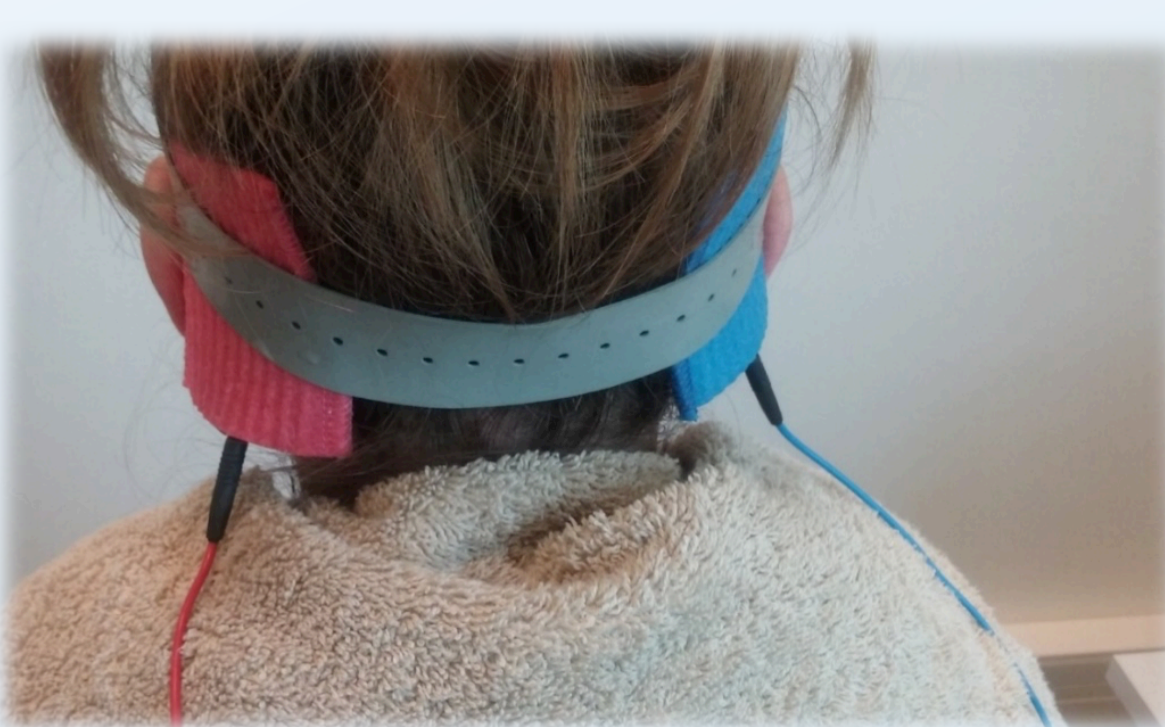

- Methods

$\checkmark$ Stimulation : 20 minutes of stimulation (NeuroConn ${ }^{\circledR}$, Germany) between 4 and 6 p.m. The intensity was $1000 \mu \mathrm{A}$ and the polarity was changed four times during each session (figure 1)

$\checkmark$ Mesures : To evaluate mood, participants completed Positive Affect and Negative Affect Schedule (PANAS) and Beck Depression Inventory (BDI) scales before and after GVS treatment.

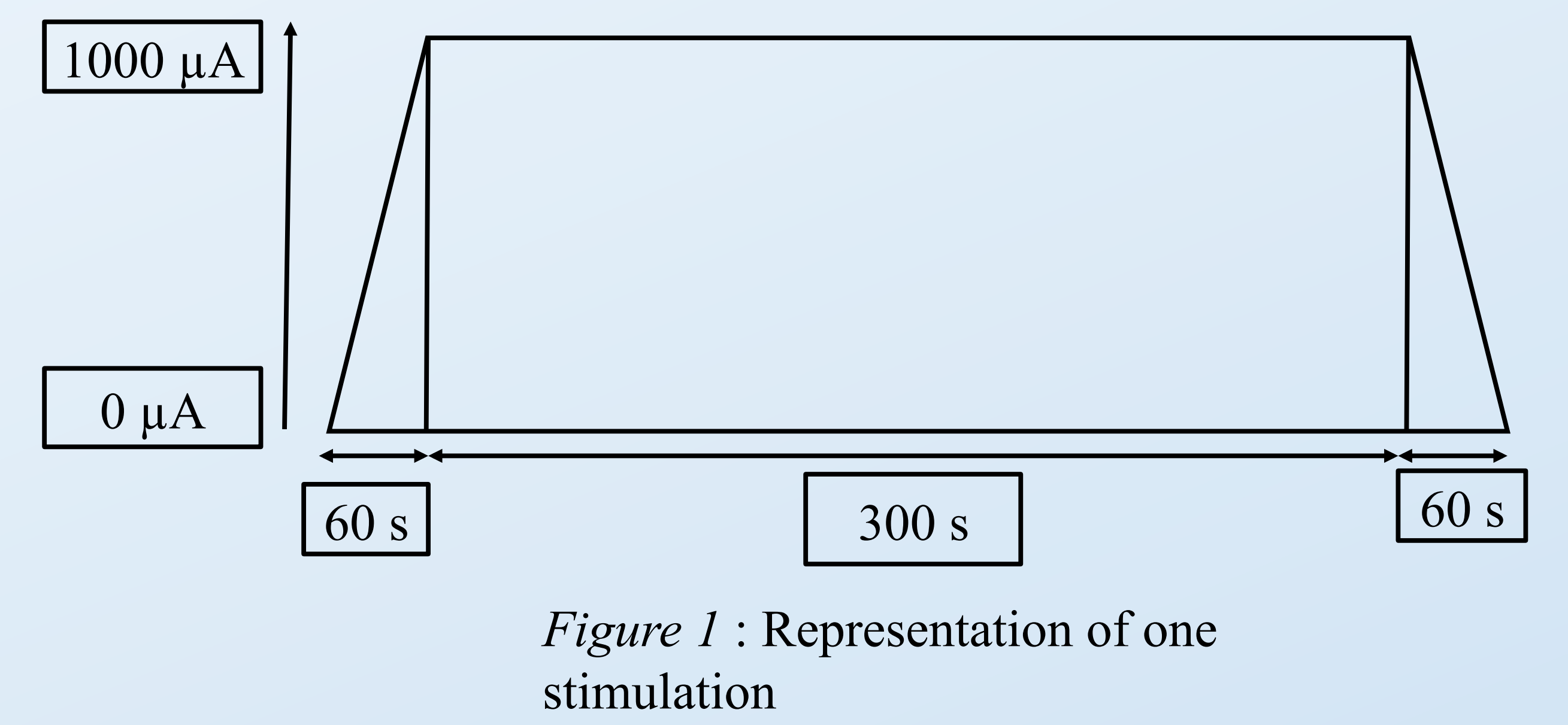

- Design : Pre-test

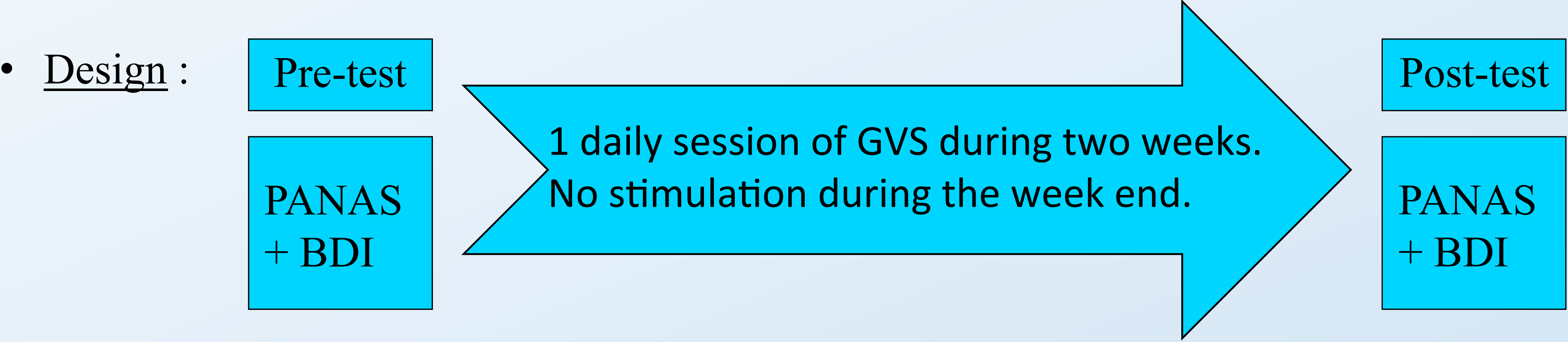

Post-test stimulation

\section{Preliminary results}

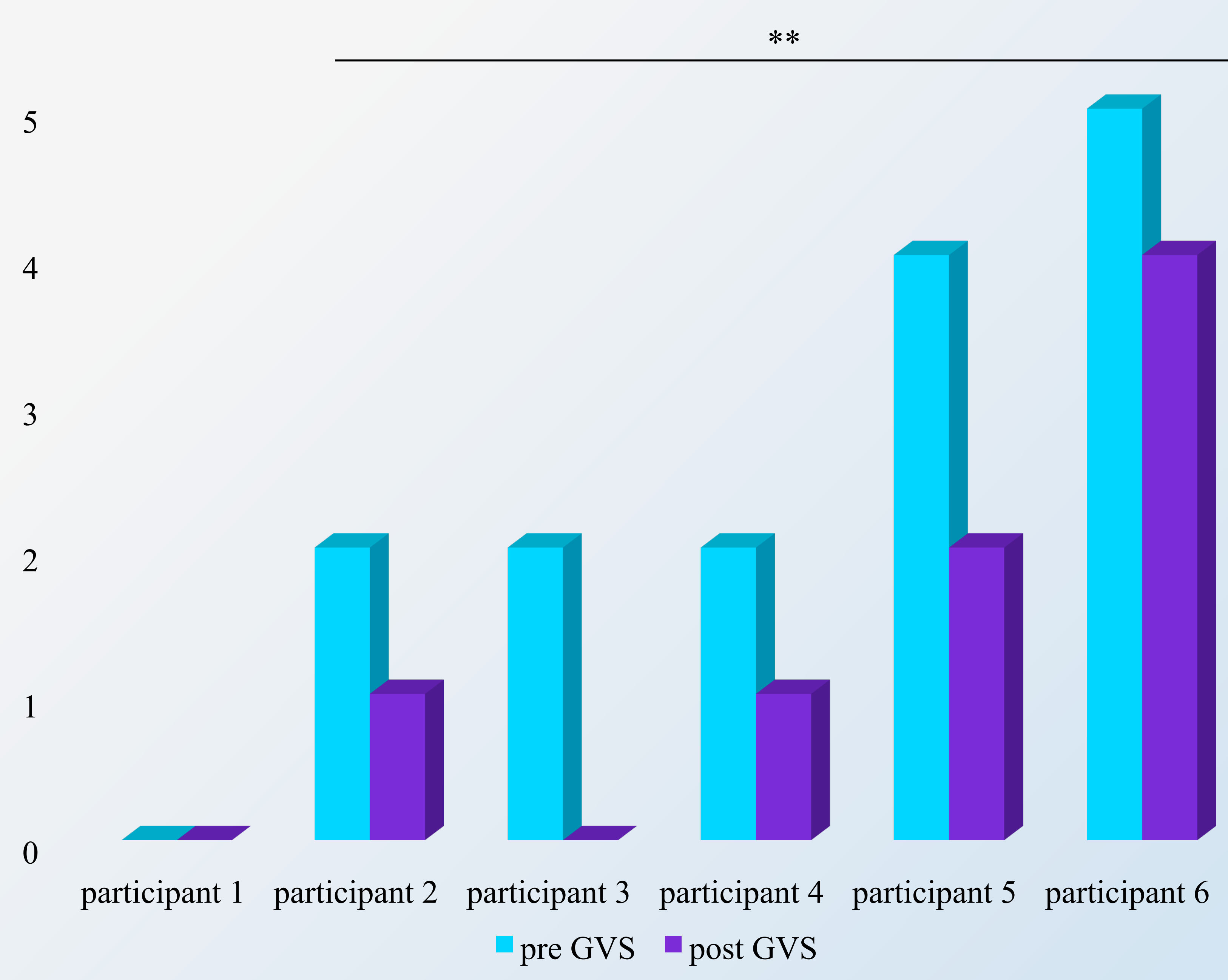

Figure 2 : Representation of BDI score before and after GVS program for each participant. $* *: \mathrm{p}<0,05$.

Diminution of BDI score after GVS program.

\section{Discussion and perspective}

We observed a significant improvement of BDI score after GVS, but in the literature it is typically noted that BDI score in test and re-test is improved. Our preliminary study did not include a control group. Currently we have a similar study with GVS. This study includes a control group to confirm the impact of GVS on mood and biological rhythms in healthy young population.

This study is the first step of a larger project on rehabilitation of biological rhythms. The goal of this first step is to confirm the effect of GVS on mood because biological rhythms and mood are linked. The literature report that sleep/wake cycle disturbances can induce mood alterations.

Our objective is to develop a rehabilitation program for people with biological rhythms disorders (sleep disorders) using vestibular stimulations (GVS and vestibular rotatory chair).

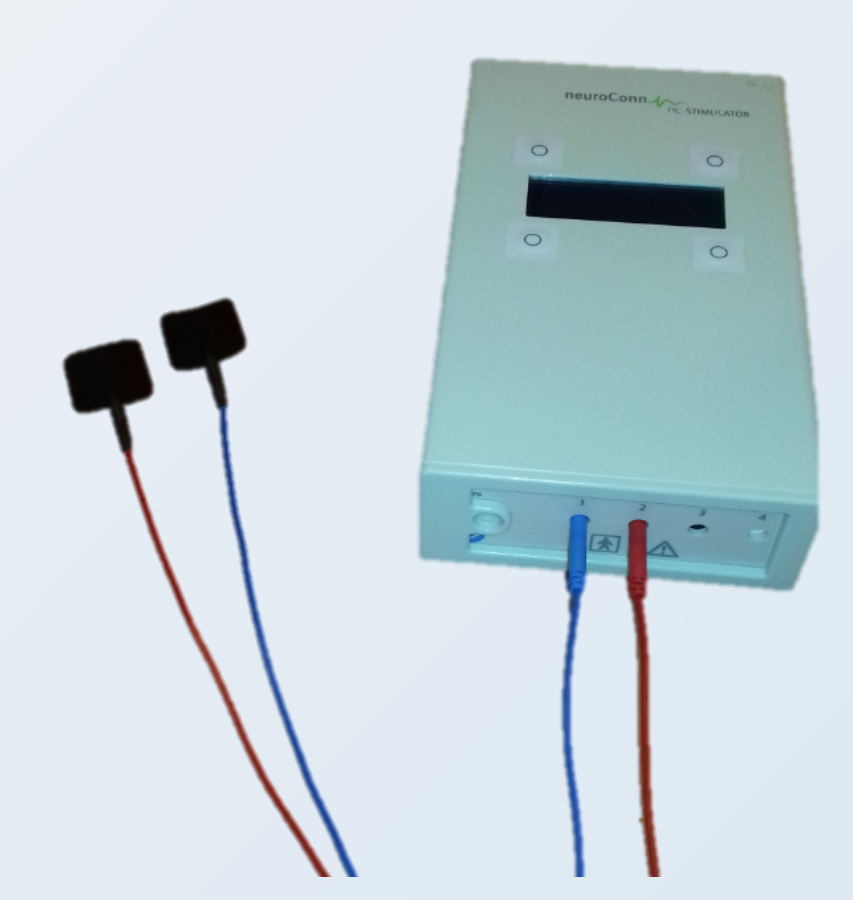

Galvanic vestibular stimulation

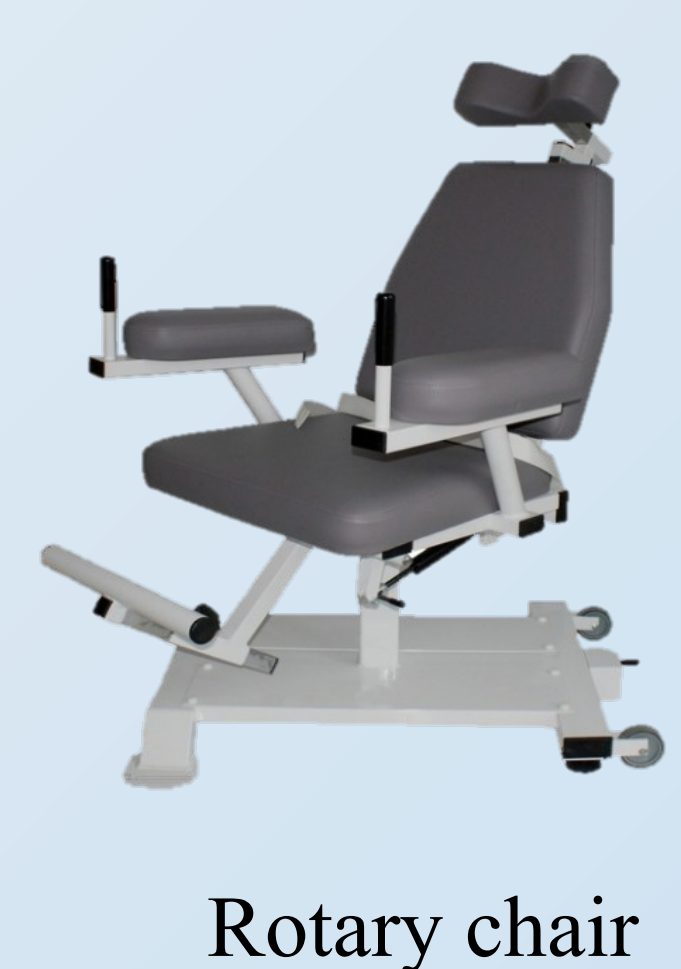

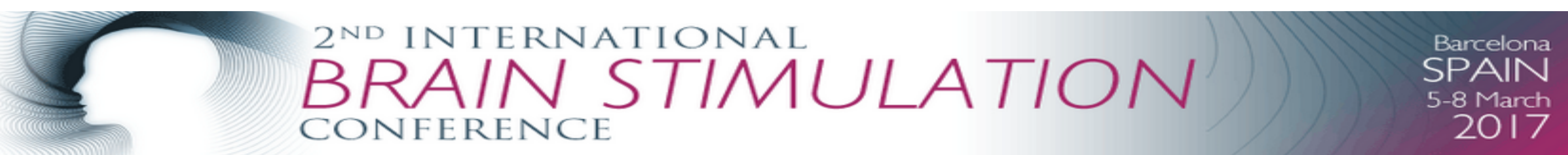

